\title{
25.
}

\section{MÉMOIRE SUR LES FONCTIONS DOUBLEMENT PÉRIODIQUES.}

[From the Journal des Mathématiques (Liouville), tom. x. (1845), pp. 385-420.]

Un des plus beaux résultats des recherches de l'illustre Abel, dans la théorie des fonctions elliptiques, consiste dans les expressions qu'il obtint pour les fonctions inverses $\phi \alpha, f \alpha, F \alpha$ (équivalentes à-peu-près à $\sin a m \alpha, \cos a m \alpha, \Delta a m \alpha$ ) en forme de fractions avec un dénominateur commun: ce dénominateur et les trois numérateurs étant chacun le produit d'une suite infinie double de facteurs. On ne sait pas à quel point Abel avait poussé l'investigation des propriétés de ces nouvelles fonctions; on trouve seulement, dans une Lettre à Legendre, imprimée parmi ses (Euvres [t. II. p. 259, Ed. 2, p. 274], qu'il s'en était occupé. Depuis, les fonctions $H, \Theta$, qui sont essentiellement les mêmes que ces fonctions d'Abel, ont été l'objet des savantes recherches de M. Jacobi, à qui l'on doit, en particulier, la belle formule

$$
\log \Theta(\alpha)-\log \Theta(0)=\frac{1}{2} \alpha^{2}\left(1-\frac{E_{\gamma}}{K}\right)-k^{2} \int_{0}^{a} d \alpha \int_{0}^{\alpha} d \alpha \sin ^{2} a m \alpha,
$$

qui est vraiment fondamentale, et sur laquelle on peut dire que sa théorie est basée. Mais les expressions qu'obtient M. Jacobi pour les fonctions $\mathrm{H}$, $\Theta$, sont sous la forme d'un produit d'une suite infinie simple de facteurs, ce qui ne met pas à beaucoup près si bien en évidence la vraie nature de ces fonctions que les expressions d'Abel; celles-ci sont, en outre, si analogues aux formules en produits infinis des fonctions circulaires, que l'on est seulement étonné que personne ne se soit avisé jusqu'ici de les poser, à priori, comme les définitions les plus simples des fonctions doublement périodiques, pour en déduire la théorie de ces fonctions. C'est de cette manière que je me propose de traiter ici la question. Je prends pour définitions les formules d'Abel, en supposant, pour plus de généralité, que les fonctions complètes $\Omega, \Upsilon\left(K, K^{\prime}\right.$ de M. Jacobi) sont chacune de la forme $A+B \sqrt{-1}$ (ce qui donne lieu à quelques intégrations assez délicates). Et de ces seules équations, sans me servir en rien de la 
théorie des fonctions elliptiques, je déduis les propriétés fondamentales des fonctions en question, et de là des fonctions elliptiques. On a ainsi quatre fonctions à considérer au lieu des deux $\mathrm{H}, \Theta$, dont l'une est pour ainsi dire analogue à un sinus et les autres à des cosinus. Mais ce qu'il y a de remarquable, c'est l'apparition d'un facteur exponentiel qui entre presque partout. On le prévoyait d'après les formules de M. Jacobi, mais ces formules n'expliquent pas, ce me semble, pourquoi ce facteur s'y rencontre: mon analyse le fait voir de la manière la plus satisfaisante. Ce facteur résulte, en effet, de ce que, pour les produits infinis doubles, il ne suffit pas, pour obtenir un résultat déterminé, d'attribuer aux deux entiers variables des valeurs quelconques depuis $-\infty$ jusqu'à $\infty$, même en supposant l'égalité des valeurs positives et négatives; il faut, en outre, établir une relation entre les valeurs infinies que reçoivent les deux variables. Mais dans les produits que je considère, on démontre qu'en supposant toujours cette égalité des valeurs positives et négatives, quelque liaison que l'on établisse entre les valeurs infinies, il résulte toujours la même valeur du produit, à un facteur exponentiel près, dont l'indice est le carré de $x$, multiplié par une constante dont la valeur s'exprime au moyen d'une intégrale définie double, et qui dépend de la liaison établie entre les valeurs infinies des variables. C'est-à-dire qu'en multipliant par un facteur exponentiel de cette forme, convenablement choisi, on peut changer à volonté la relation en question sans affecter la valeur du produit. Voilà l'idée fondamentale du Mémoire qui suit.

En me servant de quelques formules de M. Cauchy, relatives à la décomposition des fonctions en fractions simples, j'établis d'une manière rigoureuse des relations entre les trois quotients de mes quatre fonctions, qui sont les mêmes par lesquelles Abel démontre les propriétés fondamentales des fonctions $\phi, f, F$. Ces formules une fois obtenues, on peut supposer connue toute la théorie des fonctions elliptiques. Ces théorèmes de $\mathrm{M}$. Cauchy me conduisent, en outre, à un grand nombre de nouvelles formules qui contiennent des suites infinies doubles. Parmi selles-ci, il y en a une qui me fournit la démonstration du théorème déjà cité de M. Jacobi, théorème duquel il déduit une foule de résultats intéressants. Je finis en citant ceux qui se rapportent de plus près aux fonctions dont je parle. J'espère reprendre une autre fois la considération d'une autre partie de la théorie, dans laquelle j'entrevois des conclusions intéressantes.

Soient $\Omega, \Upsilon$ des quantités finies quelconques, assujetties à la seule condition que la fraction $\Omega: \Upsilon$ ne soit pas réelle. En représentant par $m, n$ des entiers positifs ou négatifs quelconques, mettons, pour abréger,

$$
m \Omega+n \Upsilon=(m, n)
$$

et considérons une expression de cette forme

$$
u=x \Pi\left\{1+\frac{x}{(m, n)}\right\} .
$$

où le symbole $\Pi$ dénote, comme à l'ordinaire, le produit d'un nombre infini de facteurs que l'on obtient en donnant à $m, n$ des valeurs entières quelconques, depuis $-\infty$ jusqu'à 
$+\infty$, en excluant seulement la combinaison $(m=0, n=0)$. Pour qu'une telle expression soit finie, il faut que pour chaque combinaison de valeurs de $m$, $n$, il y en ait une autre des mêmes valeurs avec les signes contraires. Cependant, comme on l'a déjà expliqué, cela ne suffit pas pour rendre déterminée la valeur de $u$. Soit $\phi$ une fonction de $m, n$ qui ne change pas en changeant à la fois les signes de ces deux quantités; et imaginons que l'équation

$$
\phi=T
$$

représente une courbe fermée, dont tous les points s'éloignent, au cas limite de $T=\infty$, d'une distance infinie de l'origine. Cela posé, en donnant à $m, n$ des valeurs entières qui satisfassent à cette condition $\phi=T$, et puis faisant $T=\infty$, on obtient pour $u$ une valeur parfaitement déterminée, qui dépend de la forme de la fonction $\phi$. Soit $u^{\prime}$ ce que devient la fonction $u$ en changeant seulement l'équation aux limites dans l'équation analogue

$$
\phi^{\prime}=T
$$

on peut, pour simplifier, supposer que la courbe représentée par cette, équation soit située entièrement en dehors de celle que représente l'équation

$$
\phi=T,
$$

mais cela n'est pas essentiel. Il est facile de trouver une relation très-simple qui existe entre ces deux expressions $u^{\prime}$ et $u$. En effet,

$$
u^{\prime}: u=\Pi\left\{1+\frac{x}{(m, n)}\right\},
$$

en donnant à $m, n$ des valeurs qui satisfassent à la fois aux deux conditions $\phi>T$, $\phi^{\prime}<T$. Donc, en considérant toujours ces valeurs,

$$
\log u^{\prime}-\log u=\log \Pi\left\{1+\frac{x}{(m, n)}\right\}=\boldsymbol{\Sigma} \log \left\{1+\frac{x}{(m, n)}\right\}=x \boldsymbol{\Sigma} \frac{1}{(m, n)}-\frac{1}{2} x^{2} \boldsymbol{\Sigma} \frac{1}{(m, n)^{2}}+\ldots
$$

Dans cette expression, les termes qui contiennent les puissances impaires $x$ s'évanouissent, à cause des valeurs égales positives et négatives. Mais puisque, à la limite, $m, n$ ne reçoivent que des valeurs infinies, on peut négliger les termes multipliés par $x^{4}$, \&c. Donc

$$
\log u^{\prime}-\log u=-\frac{1}{2} x^{2} \mathbf{\Sigma} \frac{1}{(m, n)^{2}},
$$

ou enfin,

$$
\log u^{\prime}-\log u=-\frac{1}{2} A x^{2}, \quad u^{\prime}=u \epsilon^{-\frac{1}{2} A x^{2}}
$$

où j'ai représenté par $\epsilon$ la base du système hyperbolique de logarithmes, et où $A$ est donné par l'équation

$$
A=\mathbf{\Sigma} \frac{1}{(m, n)^{2}}
$$


Il est facile de voir que l'on peut changer la sommation en intégration double, et écrire

$$
A=\iint \frac{d m d n}{(m, n)^{2}}
$$

entre les mêmes limites qu'auparavant, c'est-à-dire que $m, n$ doivent satisfaire aux deux conditions $\phi>T, \phi^{\prime}<T$.

Prenons par exemple, pour limite supérieure,

$$
\left(m^{2}=\mathrm{m}^{2}, \quad n^{2}=\mathrm{n}^{2}\right),
$$

et pour limite inférieure,

$$
\left(m^{2}+n^{2}=T^{2}\right) ;
$$

$\mathrm{m}, \mathrm{n}$ sont censés contenir $T$ comme facteur, de manière qu'ils deviennent infinis avec cette quantité; on suppose aussi $\mathrm{m}>T, \mathrm{n}>T$, mais cela est seulement pour la clarté. écrirons

Il devient nécessaire à ce point de définir de plus près les valeurs de $\Omega, \Upsilon$; nous

$$
\Omega=\omega+\omega^{\prime} i, \quad \Upsilon=v+v^{\prime} i
$$

où $i=\sqrt{-1} ; \omega, \omega^{\prime}, v, v^{\prime}$ sont des quantités réelles, telles que $\omega v^{\prime}-\omega^{\prime} v$ ne s'évanouit pas; c'est la condition pour que $\Omega: \Upsilon$ contienne une partie imaginaire.

Avec les coordonnées polaires

$$
A=\iint \frac{d r d \theta}{r(\Omega \cos \theta+\Upsilon \sin \theta)^{2}}=\int \frac{d \theta(\log r-\log T)}{(\Omega \cos \theta+\Upsilon \sin \theta)^{2}} .
$$

en représentant par $\mathbf{r}$ ce que devient $r$ à la limite supérieure; lïntégrale doit être prise depuis $\theta=0$ jusqu'à $\theta=2 \pi$. On voit tout de suite que la partie qui contient $\log T$ s'évanouit; donc

$$
A=\int_{0}^{2 \pi} \frac{\log \mathbf{r} d \theta}{(\Omega \cos \theta+\Upsilon \sin \theta)^{2}}
$$

Soit $\alpha$ un angle positif plus petit que $\frac{1}{2} \pi$, tel que $\tan \alpha=\frac{m}{n}$, on a évidemment

$$
\mathrm{r}=\frac{ \pm \mathrm{m}}{\cos \theta}
$$

depuis $\theta=-\alpha$ jusqu'à $\theta=+\alpha$, ou depuis $\theta=\pi-\alpha$ jusqu'à $\theta=\pi+\alpha$, et

$$
\mathrm{r}=\frac{ \pm \mathrm{n}}{\cos \theta}
$$

depuis $\theta=\alpha$ jusqu'à $\theta=\pi-\alpha$, ou depuis $\theta=\pi+\alpha$ jusqu'à $\theta=2 \pi-\alpha$ (le signe ambigu, de manière que $\mathrm{r}$ soit toujours positif). En réunissant les parties opposées de l'intégrale, on obtient

$$
A=2 \int_{-a}^{a} \frac{(\log m-\log \cos \theta) d \theta}{(\Omega \cos \theta+\Upsilon \sin \theta)^{2}}+2 \int_{a}^{\pi-a} \frac{(\log n-\log \sin \theta) d \theta}{(\Omega \cos \theta+\Upsilon \sin \theta)^{2}},
$$


ou, en mettant dans la seconde intégrale $\frac{1}{2} \pi-\alpha=\alpha^{\prime}$, et $\frac{1}{2} \pi-\theta$ au lieu de $\theta$,

$$
A=2 \int_{-a}^{a} \frac{(\log m-\log \cos \theta) d \theta}{(\Omega \cos \theta+\Upsilon \sin \theta)^{2}}+2 \int_{-a^{\prime}}^{a} \frac{(\log n-\log \cos \theta) d \theta}{(\Upsilon \cos \theta+\Omega \sin \theta)^{2}} .
$$

La première intégrale se réduit à

$$
\begin{aligned}
& -2(\log \mathrm{m}-\log \cos \theta) \frac{1}{\Upsilon(\Omega+\Upsilon \tan \theta)} \text { (entre les limites) } \\
& +\frac{2}{\pi} \int_{-\alpha}^{a} \frac{\tan \theta d \theta}{(\Omega+\Upsilon \tan \theta)} \\
& =\frac{4(\log m-\log \cos \alpha) \tan \alpha}{\Omega^{2}-\Upsilon^{2} \tan ^{2} \alpha}-4 \int_{0}^{a} \frac{\tan ^{2} \theta d \theta}{\Omega^{2}-\Upsilon^{2} \tan ^{2} \theta} \\
& =\frac{4(\log m-\log \cos \alpha) \tan \alpha}{\Omega^{2}-\Upsilon^{2} \tan ^{2} \alpha}+\frac{4}{\Omega^{2}+\Upsilon^{2}} \int_{0}^{a} d \theta\left[1-\frac{\Omega^{2}\left(1+\tan ^{2} \theta\right)}{\Omega^{2}-\Upsilon^{2} \tan ^{2} \theta}\right] .
\end{aligned}
$$

Liintégrale, dans cette formule

$$
\int_{0}^{a} \frac{\left(1+\tan ^{2} \theta\right) d \theta}{\Omega^{2}-\Upsilon^{2} \tan ^{2} \theta}=\int_{0}^{\tan \alpha} \frac{d x}{\Omega^{2}-\Upsilon^{2} x^{2}},
$$

s'exprime tout de suite par les logarithmes, mais il faut apporter une attention particulière à la manière de déterminer quelle valeur doit être attribuée à ce logarithme, dans le cas où la partie réelle en est négative. Je renvoie cette discussion à une Note. Voici le résultat auquel j’arrive.

En représentant par $L u$ la valeur principale du logarithme de $u$, quand il y a une valeur principale (c'est-à-dire quand la partie réelle de $u$ est positive), et écrivant

$$
L_{ \pm \pi i} u=L u \text {, ou } L(-u) \pm \pi i,
$$

selon qu'il y a une valeur principale de $\log u$, ou de $\log (-u)$, on a

$$
\int_{0}^{\tan \alpha} \frac{d x}{\Omega^{2}-\Upsilon^{2} x^{2}}=\frac{1}{2 \Omega \Upsilon} L_{ \pm_{n i}}\left(\frac{\Omega+\Upsilon \tan \alpha}{\Omega-\Upsilon \tan \alpha}\right)
$$

en prenant le signe supérieur pour $\omega v^{\prime}-\omega^{\prime} v$ positif, et le signe inférieur pour $\omega v^{\prime}-\omega^{\prime} v$ négatif.

La première partie de $A$ se réduit donc à

$$
\frac{4(\log m-\log \cos \alpha) \tan \alpha}{\Omega^{2}-\Upsilon^{2} \tan ^{2} \alpha}+\frac{4}{\Omega^{2}+\Upsilon^{2}}\left(\alpha-\frac{1}{2} \frac{\Omega}{\Upsilon} L_{ \pm \pi i} \frac{\Omega+\Upsilon \tan \alpha}{\Omega-\Upsilon \tan \alpha}\right),
$$

ou, en rétablissant la valeur de $\alpha$, à

$$
\frac{4 m n \log \sqrt{m^{2}+n^{2}}}{m^{2} \Omega^{2}-n^{2} \Upsilon^{2}}+\frac{4}{\Omega^{2}+\Upsilon^{2}}\left(\arctan \frac{n}{m}-\frac{1}{2} \frac{\Omega}{\Upsilon} L_{ \pm m i} \frac{m \Omega+n \Upsilon}{m \Omega-n \Upsilon}\right) .
$$


Pour avoir la seconde partie de $A$, il faut changer $\mathrm{m}, \mathrm{n}, \Omega, \Upsilon$ en $\mathrm{n}, \mathrm{m}, \Upsilon, \Omega$. En faisant cela, on change le signe de $\omega v^{\prime}-\omega^{\prime} v$; ainsi il faut écrire $L_{\mp \pi i}$ au lieu de $L_{ \pm \pi i}$. En réunissant les deux parties, et mettant $\frac{1}{2} \pi$ au lieu de $\operatorname{arc} \tan \frac{\mathrm{n}}{\mathrm{m}}+\arctan \frac{\mathrm{m}}{\mathrm{n}}$, on obtient enfin

$$
A=\frac{2}{\Omega^{2}+\Upsilon^{2}}\left\{\pi+\frac{\Omega}{\Upsilon} L_{ \pm \pi i}\left(\frac{\mathrm{m} \Omega+\mathrm{n} \Upsilon}{\mathrm{m} \Omega-\mathrm{n} \Upsilon}\right)-\frac{\Upsilon}{\Omega} L_{ \pm \pi i}\left(\frac{\mathrm{n} \Upsilon+\mathrm{m} \Omega}{\mathrm{n} \Upsilon-\mathrm{m} \Omega}\right)\right\}
$$

formule que l'on pourrait rendre plus simple en distinguant les deux cas où le premier ou le second logarithme a une valeur principale; mais il vaut mieux la laisser sous cette forme.

On aurait pu croire que la valeur de $A$ pourrait s'obtenir plus facilement en réduisant $A$ à la différence de deux intégrales définies, les conditions pour les limites étant données, dans la première, par $m^{2}<\mathrm{m}^{2}, n^{2}<\mathrm{n}^{2}$, et, pour la seconde, par $m^{2}+n^{2}<T^{2}$, en désignant généralement les cocrdonnées par $m, n$. Cependant, de cette manière, on admet dans les deux intégrales les valeurs $m=0, n=0$, qui rendent infinie la fonction à intégrer. Ainsi la valeur de l'intégrale dépendrait du choix des variables, et l'on obtiendrait des résultats inexacts. Autrement dit, on obtiendrait de cette façon la valeur de $A$, à une quantité $\nabla$ près, qui est la différence de deux intégrales de la même forme, entre des limites $m^{2}<\mu^{2}, n^{2}<\nu^{2}$ ou $m^{2}+n^{2}<\tau^{2}, \mu, \nu, \tau$ des quantités infiniment petites. On voit tout de suite que $\nabla$ n'est pas pour cela infiniment petit (en effet, sa valeur dépend du rapport $\mu: \nu$ et nullement des valeurs absolues de ces quantités), et, pour en trouver la valeur, il faudrait se servir de l'analyse précédente.

Soit, comme exemple, $\frac{m}{n}=\infty$,

$$
\begin{aligned}
A & =\frac{2}{\Omega^{2}+\Upsilon^{2}}\left\{\pi-\frac{\Omega}{\Upsilon} L_{ \pm \pi i}(1)-\frac{\Upsilon}{\Omega} L_{\mp \pi i}(-1)\right\} \\
& =\frac{2 \pi}{\Omega^{2}+\Upsilon^{2}}\left(1 \pm \frac{\Upsilon i}{\Omega}\right)=\frac{2 \pi}{\Omega(\Omega \mp \Upsilon i)}=-\frac{2 \pi i}{\Omega \Upsilon} \cdot \frac{\Upsilon}{\Omega \mp \Upsilon i}
\end{aligned}
$$

De même, pour $\frac{\mathrm{n}}{\mathrm{m}}=\infty$,

$$
\begin{aligned}
A & =\frac{2}{\Omega^{2}+\Upsilon^{2}}\left\{\pi-\frac{\Omega}{\Upsilon} L_{ \pm \pi i}(-1)-\frac{\Pi}{\Upsilon} L_{\mp \pi i}(1)\right\} \\
& =\frac{2 \pi}{\Omega^{2}+\Upsilon^{2}}\left(1 \mp \frac{\Omega i}{\Upsilon}\right)= \pm \frac{2 \pi i}{\Upsilon(\Omega \mp \Upsilon)}=\mp \frac{2 \pi i}{\Omega \Upsilon} \cdot \frac{\Omega}{\Omega \mp \Upsilon i} .
\end{aligned}
$$

En représentant par $A^{\prime}, A^{\prime \prime}$ ces deux valeurs de $A$, on a

$$
A^{\prime}-A^{\prime \prime}=\frac{\mp 2 \pi i}{\Omega \Upsilon}=-26
$$

où j’écris

$$
6= \pm \frac{\pi i}{\Omega \Upsilon}
$$

c. 
en prenant toujours le signe supérieur pour $\omega v^{\prime}-\omega^{\prime} v$ positif, et le signe inférieur pour $\omega v^{\prime}-\omega^{\prime} v$ négatif.

Soient $u_{6}$ ce que devient $u$ en prenant $\frac{m}{n}=\infty, u_{-}$ce que devient cette même fonction en prenant $\frac{\mathrm{n}}{\mathrm{m}}=\infty$; on a évidemment

$$
u_{-6}: u_{6}=\epsilon^{-\frac{1}{2}\left(A^{\prime}-A^{\prime \prime}\right) x^{2}}=\epsilon^{6 x^{2}} .
$$

On peut donc s'imaginer une fonction $U$ donnée par les équations

$$
U=\epsilon^{-16 x^{2}} u_{-6}=\epsilon^{36 x^{2}} u_{6}
$$

On verra dans la suite que $u_{6}$ est analogue à la fonction $H(u)$ de M. Jacobi. Il convient cependant, pour la symétrie, de considérer, au lieu de $u_{6}$, la nouvelle fonction $U$ qui vient d'être définie. Quoique suffisamment donnée par ces équations, nous allons en chercher encore une autre définition. Pour cela, il faut trouver la valeur de l'intégrale définie qui détermine $A$, prise depuis la même limite inférieure jusqu'à celle donnée par l'équation

$$
\bmod (m, n)=T
$$

Mettons, comme auparavant,

$$
m=r \cos \theta, \quad n=r \sin \theta ;
$$

soient aussi

$$
\Omega_{1}=\omega-\omega^{\prime} i, \quad \Upsilon_{1}=v-v^{\prime} i .
$$

L'équation pour la limite supérieure se réduit à

$$
r^{2}(\Omega \cos \theta+\Upsilon \sin \theta)\left(\Omega_{1} \cos \theta+\Upsilon_{1} \sin \theta\right)=T^{2},
$$

et celle pour la limite inférieure, à

$$
r=T \text {. }
$$

On trouve tout de suite

$$
\begin{aligned}
A= & -\frac{1}{2} \int_{0}^{2 \pi} \frac{\log (\Omega \cos \theta+\Upsilon \sin \theta)\left(\Omega_{1} \cos \theta+\Upsilon_{1} \sin \theta\right) d \theta}{(\Omega \cos \theta+\Upsilon \sin \theta)^{2}} \\
= & \frac{1}{2 \Upsilon} \frac{1}{(\Omega+\Upsilon \tan \theta)} \log (\Omega \cos \theta+\Upsilon \sin \theta)\left(\Omega_{1} \cos \theta+\Upsilon_{1} \sin \theta\right)(\text { entre } \theta=0, \theta=2 \pi) \\
& -\frac{1}{2 \Upsilon} \int_{0}^{2 \pi} \frac{d \theta}{\Omega+\Upsilon \tan \theta}\left(\frac{\Upsilon-\Omega \tan \theta}{\Omega+\Upsilon \tan \theta}+\frac{\Upsilon_{1}-\Omega_{1} \tan \theta}{\Omega_{1}+\Upsilon_{1} \tan \theta}\right) \\
= & -\frac{1}{\Upsilon} \int_{-\frac{1}{2} \pi}^{\frac{1}{2} \pi} \frac{d \theta}{\Omega+\Upsilon \tan \theta}\left(\frac{\Upsilon-\Omega \tan \theta}{\Omega+\Upsilon \tan \theta}+\frac{\Upsilon_{1}-\Omega_{1} \tan \theta}{\Omega_{1}+\Upsilon_{1} \tan \theta}\right)
\end{aligned}
$$


D'abord

$$
\begin{gathered}
\int_{-\frac{1}{2} \pi}^{\frac{1}{2} \pi} \frac{d \theta}{\Omega+\Upsilon \tan \theta} \frac{\Upsilon_{1}-\Omega_{1} \tan \theta}{\Omega_{1}+\Upsilon_{1} \tan \theta}=\int_{-\frac{1}{2} \pi}^{\frac{1}{2} \pi}\left(\frac{M}{\Omega+\Upsilon \tan \theta}+\frac{M_{1}}{\Omega_{1}+\Upsilon_{1} \tan \theta}\right) d \theta \\
\int_{-\frac{1}{2} \pi}^{\frac{1}{2} \pi} \frac{d \theta}{\Omega+\Upsilon \tan \theta}=2 \Omega \int_{0}^{\frac{1}{2} \pi} \frac{d \theta}{\Omega^{2}-\Upsilon^{2} \tan ^{2} \theta}=\frac{2 \Omega}{\Omega^{2}+\Upsilon^{2}} \int_{0}^{\frac{1}{2} \pi}\left[1+\frac{\Upsilon^{2}\left(1+\tan ^{2} \theta\right)}{\Omega^{2}-\Upsilon^{2} \tan ^{2} \theta}\right] d \theta \\
=\frac{2 \Omega}{\Omega^{2}+\Upsilon^{2}}\left\{\theta+\frac{\Upsilon}{2 \Omega} L_{ \pm \pi i}\left(\frac{\Omega+\Upsilon \tan \theta}{\Omega-\Upsilon \tan \theta}\right)\right\} \quad\left(\text { entre } \theta=0, \quad \theta=\frac{1}{2} \pi\right) \\
=\frac{2 \Omega}{\Omega^{2}+\Upsilon^{2}}\left(\frac{1}{2} \pi \pm \frac{\Upsilon}{2 \Omega} \pi i\right)=\frac{\pi}{\Omega \mp \Upsilon i} .
\end{gathered}
$$

On a de même, en remarquant qu'en changeant $\Omega, \Upsilon$ en $\Upsilon, \Omega$, on change le signe de $\omega v^{\prime}-\omega^{\prime} v$,

$$
\int_{-\frac{1}{2} \pi}^{\frac{1}{2} \pi} \frac{d \theta}{\Omega_{1}+\Upsilon_{1} \tan \theta}=\frac{\pi}{\Omega_{1} \pm \Upsilon_{1} i}
$$

D'un autre côté,

$$
M=\frac{\Upsilon \Upsilon_{1}+\Omega_{1} \Omega_{1}}{\Upsilon \Omega_{1}-\Upsilon_{1} \Omega}, \quad M_{1}=-\frac{\Upsilon_{1}^{2}+\Omega_{1}^{2}}{\Upsilon \Omega_{1}-\Upsilon_{1} \Omega}
$$

cela donne

$$
\begin{aligned}
& \int_{-\frac{1}{2} \pi}^{\frac{1}{2} \pi} \frac{d \theta}{\Omega+\Upsilon \tan \theta} \frac{\Upsilon_{1}-\Omega_{1} \tan \theta}{\Omega_{1}+\Upsilon_{1} \tan \theta}=\frac{\pi}{\Upsilon \Omega_{1}-\Upsilon_{1} \Omega}\left\{\frac{\Upsilon \Upsilon_{1}+\Omega_{1}}{\Omega \mp \Omega_{i}}-\left(\Omega \mp \Upsilon_{i}\right)\right\} \\
& =\frac{\pi}{\Upsilon \Omega_{1}-\Upsilon_{1} \Omega} \frac{\Upsilon \Upsilon_{1}+\Omega \Omega_{1}-\left(\Omega \mp \Upsilon_{i}\right)\left(\Omega_{1} \pm \Upsilon_{1} i \mp 2 \Upsilon_{1} i\right)}{\Omega \mp \Upsilon_{i}}= \pm \frac{\pi i}{\Omega \mp \Upsilon_{i}} \pm \frac{2 \Upsilon_{1} \pi i}{\Upsilon \Omega_{1}-\Upsilon_{1} \Omega},
\end{aligned}
$$

ou enfin

$$
\int_{-\frac{1}{2} \pi}^{\frac{1}{2} \pi} \frac{d \theta}{\Omega+\Upsilon \tan \theta} \frac{\Upsilon_{1}-\Omega_{1} \tan \theta}{\Omega_{1}+\Upsilon_{1} \tan \theta}= \pm \frac{\pi i}{\Omega \mp \Upsilon i} \pm \frac{\pi \Upsilon_{1}}{\omega v^{\prime}-\omega^{\prime} v}
$$

et de même

$$
\int_{-\frac{1}{2} \pi}^{\frac{1}{2} \pi} \frac{d \theta(\Upsilon-\Omega \tan \theta)}{(\Omega+\Upsilon \tan \theta)^{2}}=\frac{ \pm \pi i}{\Omega \mp \Upsilon i}
$$

en omettant seulement le dernier terme de l'autre intégrale; ce que l'on peut vérifier, au reste, en différentiant par rapport à $\Omega, \Upsilon$ l'équation

$$
\int_{-\frac{1}{2} \pi}^{\frac{1}{2} \pi} \frac{d \theta}{\Omega+\Upsilon \tan \theta}=\frac{\pi}{\Omega \mp \Upsilon i} .
$$


On a donc, en ajoutant les deux parties qui composent l'intégrale,

$$
A=\mp \frac{2 \pi i}{\Omega \Upsilon} \frac{\Omega}{\Omega \mp \Upsilon i} \mp \frac{\Upsilon_{1} \pi}{\Upsilon\left(\omega v^{\prime}-\omega^{\prime} v\right)}
$$

Il est facile de voir, en écrivant

$$
A=\mp \frac{2 \pi i}{\Omega \Upsilon} \frac{\Omega}{\Omega \mp \Upsilon i} \pm \frac{\pi \Upsilon_{1} i}{\Upsilon\left(\Upsilon \Omega_{1}-\Upsilon_{1} \Omega\right)},
$$

que cette expression ne change pas de valeur en mettant $\Omega_{1}, \Upsilon_{1}, \Omega, \Upsilon$ au lieu de $\Omega, \Upsilon, \Omega_{1}, \Upsilon_{1}$, pourvu qu'on change aussi le signe de $i$; cela doit évidemment être ainsi et peut servir de vérification.

Soit, pour un moment, $u_{1}$ ce que devient $u$ en prenant pour limite l'équation

$$
m^{2}+n^{2}=T^{2},
$$

et supposons que dans la fonction $u$ on ait pour limite l'équation

$$
\bmod (m, n)=T \text {. }
$$

En retenant la valeur de $A$, qui vient d'être trouvée,

$$
u=\epsilon^{-\frac{1}{2} A x^{2}} \cdot u_{1} ;
$$

mais aussi

$$
U=\epsilon^{\mp \frac{1}{2} \frac{\pi i}{\Omega Y}} \cdot x^{2} u_{-6}=\epsilon\left(\mp \frac{1}{2} \frac{\pi i}{\Omega Y} \pm \frac{\pi i}{\Omega Y} \frac{\Omega}{\Omega \neq Y_{i}}\right) x^{2} u_{1},
$$

à cause de l'équation, conséquence facile des résultats précédents,

$$
u_{-6}=\epsilon^{ \pm \frac{\pi i}{\Omega Y} \frac{\Omega}{\Omega \mp Y i} x^{2}} u_{1} .
$$

En éliminant $u_{1}$, on obtient, entre $u, U$, une équation de la forme

$$
U=\epsilon^{-\frac{1}{2} B x^{2}} u \text {. }
$$

dans laquelle

$$
\begin{aligned}
B & =-A \pm \frac{\pi i}{\Omega \Upsilon} \mp \frac{2 \pi i}{\Omega \mp \Upsilon i}= \pm \frac{\pi i}{\Omega \Upsilon} \pm \frac{\Upsilon_{1} \pi}{\Upsilon\left(\omega v^{\prime}-\omega^{\prime} v\right)} \\
& =\frac{ \pm \pi}{\Omega \Upsilon\left(\omega v^{\prime}-\omega^{\prime} v\right)}\left[2\left(\omega v^{\prime}-\omega^{\prime} v\right)+\left(\omega+\omega^{\prime} i\right)\left(v-v^{\prime} i\right)\right]
\end{aligned}
$$

ou enfin

$$
B=\frac{ \pm \pi\left(\omega v+\omega^{\prime} v^{\prime}\right)}{\Omega \Upsilon\left(\omega v^{\prime}-\omega^{\prime} v\right)}=\frac{\pi\left(\omega v^{\prime}+\omega^{\prime} v^{\prime}\right)}{\Omega \Upsilon \bmod \left(\omega v^{\prime}-\omega^{\prime} v\right)}
$$


En rassemblant tous ces résultats, on a le système de formules

(A)

$$
\begin{aligned}
& \left\{\begin{array}{l}
U=\epsilon^{-\frac{1}{2} B x^{2}} u=\epsilon^{-\frac{1}{2} b x^{2}} u_{-8}=\epsilon^{\frac{1}{2}} \varepsilon x^{2} u_{6}, \\
u=\epsilon^{\frac{1}{2}(B-6) x^{2}} u_{-8}=\epsilon^{\frac{1}{2}(B+8)} x^{2} u_{6}, \\
6=\frac{\pi i}{\Omega \Upsilon} \frac{\left(\omega v^{\prime}-\omega^{\prime} v\right)}{\bmod \left(\omega v^{\prime}-\omega^{\prime} v\right)}, \\
B=\frac{\pi\left(\omega v+\omega^{\prime} v^{\prime}\right)}{\bmod \left(\omega v^{\prime}-\omega^{\prime} v\right)}, \\
u, u_{6}, u_{-6} \text { des fonctions de la forme } \\
x \Pi\left\{1+\frac{x}{(m, n)}\right\},
\end{array}\right. \\
& \begin{array}{ll}
m^{2}=\mathrm{m}^{2}, n^{2}=\mathrm{n}^{2}, & \mathrm{~m}=\infty, \mathrm{n}=\infty, \frac{\mathrm{m}}{\mathrm{n}}=\infty, \operatorname{pour} u_{\beta} ; \\
m^{2}=\mathrm{m}^{2}, n^{2}=\mathrm{n}^{2}, & m=\infty, \quad \mathrm{n}=\infty, \frac{\mathrm{n}}{\mathrm{m}}=\infty, \operatorname{pour} u_{-8} .
\end{array}
\end{aligned}
$$

A présent, il importe de remarquer que la fonction $u_{6}$ est périodique à l'égard de $\Omega$, de la manière d'un sinus ou d'un cosinus, mais ne l'est pas à l'égard de $\Upsilon$; de même $u_{-\zeta}$ est périodique à l'égard de $\Upsilon$, mais non à l'égard de $\Omega$. Quant à $U, u$, elles ne sont simplement périodiques ni à l'égard de $\Omega$, ni à l'égard de $\Upsilon$. Pour démontrer cela, considérons, par exemple, l'équation

$$
u_{6}=x \Pi\left(1+\frac{x}{(m, n)}\right)
$$

[ $m$ depuis $-m$ jusqu'à $m, n$ depuis $-n$ jusqu'à $n, m=\infty, n=\infty, \frac{m}{n}=\infty$, le système $(m=0, n=0)$ toujours exclu]; en représentant par $u_{\boldsymbol{B}}^{\prime}$ ce que devient $u_{\boldsymbol{B}}$ quand on écrit $x+\Omega$ au lieu de $x$, on a évidemment

$$
\begin{aligned}
u_{\mathbf{B}}^{\prime} & =(x+\Omega) \Pi\left(1+\frac{x+\Omega}{(m, n)}\right) \\
& =-x \Pi\left(1+\frac{1}{(m+1, n)}\right): \Pi \frac{(m+1, n)}{(m, n)},
\end{aligned}
$$

en admettant dans le premier produit la combinaison $(m=0, n=0)$, mais excluant $(m+1=0, n=0)$, et excluant l'une et l'autre du second produit. Cette équation est de la forme

$$
u_{6}^{\prime}=A^{\prime} x \Pi\left(1+\frac{x}{(m+1, n)}\right),
$$

avec les mêmes limites que dans $u$; donc

$$
u_{B}^{\prime}: u_{b}=A^{\prime} \Pi\left(1+\frac{x}{(\mathrm{~m}+1, n)}\right): \Pi\left(1+\frac{x}{(-\mathrm{m}, n)}\right),
$$


où II se rapporte à la seule variable $n$ qui doit s'étendre depuis $-\mathrm{n}$ jusqu'à $\mathrm{n}$. Puisqu'ainsi $n$ ne reçoit jamais des valeurs qui soient comparables à $\mathrm{m}$, chacun de ces produits se réduit à l'unité, et l'on obtient

$$
u_{6}^{\prime}: u_{6}=A^{\prime} \text {, }
$$

ou enfin

$$
u_{6}^{\prime}=-u_{6}
$$

en posant

$$
x=-\frac{1}{2} \Omega,
$$

et remarquant que $u$ est fonction impaire de $x$, ce qui donne

$$
A^{\prime}=-1 \text {. }
$$

Soit de même $u_{6}^{\prime \prime}$ ce que devient $u_{6}$ en changeant $x$ en $x+\Upsilon$. On a pareillement

$$
u_{6}^{\prime \prime}: u_{6}=A^{\prime \prime} \Pi\left(1+\frac{x}{(m, \mathrm{n}+1)}\right): \Pi\left(1+\frac{x}{(m,-\mathrm{n})}\right),
$$

où $\Pi$ se rapporte à la seule variable $m$. Mais ici les produits ne se réduisent point à l'unité; en effet,

$$
\begin{aligned}
& \Pi\left(1+\frac{x}{(m, \mathrm{n}+1)}\right) \text { ou } \Pi\left(1+\frac{x}{(m, \mathrm{n})}\right) \\
= & (x+\mathrm{n} \Upsilon) \Pi\left(1+\frac{x-\mathrm{n} \Upsilon}{m \Omega}\right): \mathrm{n} \Upsilon \Pi\left(1+\frac{\mathrm{n} \Upsilon}{m \Omega}\right) \\
= & \sin \frac{\pi}{\Omega}(x+\mathrm{n} \Upsilon): \sin \frac{\pi}{\Omega} \mathrm{n} \Upsilon .
\end{aligned}
$$

Mais quand la partie imaginaire de $\theta$ est infinie, on trouve

$$
\sin \theta=\frac{1}{2 i}\left(\epsilon^{\theta i}-\epsilon^{-\theta i}\right)= \pm \frac{1}{2 i} \epsilon^{ \pm \theta i},
$$

selon que la partie réelle de $\theta i$ est positive ou négative. Or la partie réelle de $\frac{i \Upsilon}{\Omega}$ est de signe contraire à $\omega v^{\prime}-\omega^{\prime} v$; on a donc

$$
\Pi\left(1+\frac{x}{(m, \mathrm{n}+1)}\right)=\epsilon^{\mp \frac{\pi i}{\Omega} x}=\epsilon^{-\mathrm{GY} x} ;
$$

de même,

$$
\Pi\left(1+\frac{x}{(m,-\mathrm{n})}\right)=\epsilon^{\mathrm{g \Upsilon x} x},
$$

et de là

$$
u_{6}^{\prime \prime}=A^{\prime \prime} \epsilon^{-28 Y x} u_{6}
$$

équation de la même forme que celle que l'on obtiendrait en posant

$$
u_{6}=\epsilon^{-6 x^{2}} u_{-6},
$$


et remarquant que $u_{-6}$ est périodique à l'égard de $\Upsilon$. On voit aussi qu'en sommant par rapport à $m$, il est permis d'exprimer $u_{b}$ par un produit infini simple, dont chaque facteur est de la forme

$$
\sin \frac{\pi}{\Omega}(x+n \Upsilon),
$$

mais qu'on n'arrive pas à un tel résultat, en sommant par rapport à $n$. En effet, l'équation

$$
u_{6}=\epsilon^{-6 x^{2}} u_{-6}
$$

fait voir que $u_{6}$ s'exprime par un tel produit où chaque terme est de la forme

$$
\sin \frac{\pi}{\Upsilon}(x+m \Omega),
$$

multiplié par le facteur exponentiel $\epsilon^{-6 x^{2}}$. On établit de cette façon l'identité, à un facteur constant près, de $u_{6}$ à $H(u)$; mais, à présent, pour éviter les longueurs, je ne me propose de considérer aucun de ces produits infinis simples.

Il faut maintenant, pour le développement de la théorie, introduire les trois fonctions qui correspondent au cosinus. Mettant, pour abréger,

j'écris

$$
\bar{m}=m+\frac{1}{2}, \quad \bar{n}=n+\frac{1}{2}
$$

Limites.

(B)

$$
\begin{cases}\mathrm{y} x=\epsilon^{-\frac{1}{2} B x^{2}} x \Pi\left\{1+\frac{x}{(m, n)}\right\} . & \bmod (m, n)=T, T=\infty . \\ \mathrm{g} x=\epsilon^{-\frac{1}{b} B x^{2}} \Pi\left\{1+\frac{x}{(\bar{m}, n)}\right\} . & \bmod (\bar{m}, n)=T . \\ G x=\epsilon^{-\frac{1}{2} B x^{2}} \Pi\left\{1+\frac{x}{(m, \bar{n})}\right\} . & \bmod (m, \bar{n})=T . \\ Z x=\epsilon^{-\frac{1}{2} B x^{2}} \Pi\left\{1+\frac{x}{(\bar{m}, \bar{n})}\right\} . & \bmod (\bar{m}, \bar{n})=T .\end{cases}
$$

En représentant par $\mathrm{y}_{6} x, \mathrm{y}_{-6} x$, \&c., ce que deviennent ces fonctions, et prenant pour limites

$$
\begin{array}{ll}
\left(m^{2}=\mathrm{m}^{2}, n^{2}=\mathrm{n}^{2}\right), & \left(\bar{m}^{2}=\overline{\mathrm{m}}^{2}, n^{2}=\mathrm{n}^{2}\right), \\
\left(m^{2}=\mathrm{m}^{2}, \bar{n}^{2}=\overline{\mathrm{n}}^{2}\right), & \left(\bar{m}^{2}=\overline{\mathrm{m}}^{2}, \bar{n}^{2}=\overline{\mathrm{n}}^{2}\right),
\end{array}
$$

où

$$
\frac{\mathrm{m}}{\mathrm{n}}=\infty \text { pour } \mathrm{y}_{6} x, \& \mathrm{c} ., \frac{\mathrm{n}}{\mathrm{m}}=\infty \text { pour } \mathrm{y}_{-6} x, \& \mathrm{c} .,
$$

on a, en général, en mettant $J$ au lieu de l'une quelconque des lettres $\mathrm{y}, \mathrm{g}, G, Z$,

$$
J_{x}=\epsilon^{\frac{1}{2} \varepsilon x^{2}} J_{6} x=\epsilon^{-\frac{1}{2} \varepsilon x^{2}} J_{-6} x .
$$

où $J_{6} x$ est périodique à l'égard de $\Omega$, et $J_{-6} x$ à l'égard de $\Upsilon$. C'est cette équation remarquable qui définit la loi de périodicité des fonctions $J$, et de laquelle se déduisent presque toutes les propriétés de ces fonctions. 
Il est clair qu'en changeant entre elles les quantités $\Omega, \Upsilon$ (quantités que nous désignerons comme les fonctions complètes), on ne change pas les fonctions $\mathrm{y} x, Z x$, tandis que gx se change en $G x$, et $G x$ en gx. On change de cette manière 6 en -6 et $B$ en $-B$ (par exemple, $\mathrm{y}_{\boldsymbol{6}} x$ se change en $\mathrm{y}_{-\boldsymbol{b}} x$, \&c.). Cette considération fait voir que toute propriété des fonctions $J$ est double, et donne le moyen le plus facile pour passer d'une propriété quelconque à la propriété correspondante.

On tire immédiatement des définitions mêmes les équations

$$
\left\{\begin{array}{rlrlrl}
\mathrm{y}(-x) & =-\mathrm{y} x, & \mathrm{~g}(-x) & =\mathrm{g} x, & G(-x)=G x, & Z(-x)=Z x ; \\
\mathrm{y} 0 & =0, & \mathrm{~g} 0=1, & G 0=1, & Z 0=1 ; \\
y^{\prime} 0=1 . & & & &
\end{array}\right.
$$

Il est facile de démontrer, de la même manière dont nous avons prouvé la périodicité de $J_{6} x$ à l'égard de $\Omega$, que ces fonctions se changent l'une en l'autre, à un facteur constant près, en changeant $x$ en $x+\frac{1}{2} \Omega$. Faisant donc attention à l'équation qui lie ensemble $J x$ et $J_{6} x$, on obtient le système de formules

$$
\left.\begin{array}{l}
\mathrm{y}\left(x+\frac{1}{2} \Omega\right)=\epsilon^{\frac{1}{2} \beta \Omega x} A \mathrm{~g} x, \\
\mathrm{~g}\left(x+\frac{1}{2} \Omega\right)=\epsilon^{\frac{1}{2} \beta \Omega x} B \mathrm{y} x, \\
G\left(x+\frac{1}{2} \Omega\right)=\epsilon^{\frac{1}{2} \beta \Omega x} C Z x, \\
Z\left(x+\frac{1}{2} \Omega\right)=\epsilon^{\frac{1}{2} \beta \Omega x} D Z x .
\end{array}\right\}
$$

Pour déterminer $A, B, C, D$, posons

$$
x=0 \text { ou } x=-\frac{1}{2} \Omega .
$$

En écrivant, pour abréger,

$$
\epsilon^{6 \Omega^{2}}=\epsilon^{ \pm \frac{\pi \Omega i}{Y}}=q_{1}^{-1},
$$

on trouve

$$
\begin{aligned}
& A=\mathrm{y}\left(\frac{1}{2} \Omega\right), \\
& B=-q_{1}^{-\frac{1}{2}}: \mathrm{y}\left(\frac{1}{2} \Omega\right) \\
& C=G\left(\frac{1}{2} \Omega\right)=q_{1}^{-\frac{1}{2}}: Z\left(\frac{1}{2} \Omega\right), \\
& D=Z\left(\frac{1}{2} \Omega\right)=q_{1}^{-\frac{1}{2}}: G\left(\frac{1}{2} \Omega\right)
\end{aligned}
$$

d'où l'on déduit cette équation de condition,

$$
G\left(\frac{1}{2} \Omega\right) Z\left(\frac{1}{2} \Omega\right)=q_{1}^{-\frac{1}{k}}
$$

On a de même le système

$$
\begin{aligned}
& \mathrm{y}\left(x+\frac{1}{2} \Upsilon\right)=\epsilon^{-\frac{1}{2} \mathrm{EY} x} A^{\prime} G x, \\
& \mathrm{~g}\left(x+\frac{1}{2} \Upsilon\right)=\epsilon^{-\frac{1}{2} \mathrm{~g} x} B^{\prime} Z x, \\
& G\left(x+\frac{1}{2} \Upsilon\right)=\epsilon^{-\frac{1}{2} \operatorname{EY} x} C^{\prime} \mathrm{y} x, \\
& Z\left(x+\frac{1}{2} \Upsilon\right)=\epsilon^{-\frac{1}{2} \operatorname{EY} x} D^{\prime} G x,
\end{aligned}
$$


De là, si

$$
\epsilon^{-\odot \Upsilon^{2}}=\epsilon^{ \pm \frac{\pi \Upsilon i}{\Omega}}=q^{-1}
$$

ce qui donne

$$
\log q \log q_{1}=-\pi^{2}
$$

il résulte

$$
\begin{aligned}
& A^{\prime}=\mathrm{y}\left(\frac{1}{2} \Upsilon\right) \\
& B^{\prime}=\mathrm{g}\left(\frac{1}{2} \Upsilon\right)=q^{-\frac{1}{4}}: Z\left(\frac{1}{2} \Upsilon\right) \\
& C^{\prime}=-q^{-\frac{1}{4}}: \mathrm{y}\left(\frac{1}{2} \Upsilon\right) \\
& D^{\prime}=Z\left(\frac{1}{2} \Upsilon\right): \mathrm{g}\left(\frac{1}{2} \Upsilon\right)
\end{aligned}
$$

d'où nous tirons l'équation de condition

$$
g\left(\frac{1}{2} \Upsilon\right) Z\left(\frac{1}{2} \Upsilon\right)=q^{-\frac{1}{4}}
$$

En posant $x=\frac{1}{2} \Upsilon$ dans l'équation pour $\mathrm{y}\left(x+\frac{1}{2} \Omega\right)$ et $x=\frac{1}{2} \Omega$ dans l'équation pour $\mathrm{y}\left(x+\frac{1}{2} \Upsilon\right)$, on obtient aussi

$$
\mathrm{y}\left(\frac{1}{2} \Omega\right) \mathrm{g}\left(\frac{1}{2} \Upsilon\right)=-i \mathrm{y}\left(\frac{1}{2} \Upsilon\right) G\left(\frac{1}{2} \Omega\right)
$$

et l'on déduirait cette même équation, ou une équation équivalente, des autres formules, de manière qu'on ne peut pas trouver d'autres équations de condition.

Enfin, en rapprochant ces systèmes, on obtient

$$
\left.\begin{array}{l}
\mathrm{y}\left(x+\frac{1}{2} \Omega+\frac{1}{2} \Upsilon\right)=\epsilon^{\frac{1}{2} \beta x(\Omega-\Upsilon)} A^{\prime \prime} Z x, \\
\mathrm{~g}\left(x+\frac{1}{2} \Omega+\frac{1}{2} \Upsilon\right)=\epsilon^{\frac{1}{2} \beta x(\Omega-\Upsilon)} B^{\prime \prime} \mathrm{g} x, \\
G\left(x+\frac{1}{2} \Omega+\frac{1}{2} \Upsilon\right)=\epsilon^{\frac{1}{2} \beta x(\Omega-\Upsilon)} C^{\prime \prime} G x, \\
Z\left(x+\frac{1}{2} \Omega+\frac{1}{2} \Upsilon\right)=\epsilon^{\frac{1}{2} \beta x(\Omega-\Upsilon)} D^{\prime \prime} \mathrm{y} x,
\end{array}\right\}
$$

avec les équations suivantes pour les coefficients,

$$
\left.\begin{array}{l}
A^{\prime \prime}=(-1)^{\frac{1}{2}} y\left(\frac{1}{2} \Omega\right) g\left(\frac{1}{2} \Upsilon\right), \\
B^{\prime \prime}=-(-1)^{\frac{1}{2}} q_{1}^{-\frac{1}{4}} y\left(\frac{1}{2} \Upsilon\right): y\left(\frac{1}{2} \Omega\right), \\
C^{\prime \prime}=(-1)^{\frac{1}{2}} R\left(\frac{1}{2} \Omega\right) Z\left(\frac{1}{2} \Upsilon\right), \\
D^{\prime \prime}=-(-1)^{\frac{1}{2}} q^{-\frac{1}{4}} Z\left(\frac{1}{2} \Omega\right): y\left(\frac{1}{2} \Upsilon\right) .
\end{array}\right\}
$$

En rassemblant les équations entre $J\left(\frac{1}{2} \Omega\right), J\left(\frac{1}{2} \Upsilon\right)$, on a

$$
\left.\begin{array}{rl}
g\left(\frac{1}{2} \Omega\right) & =0, \\
G\left(\frac{1}{2} \Upsilon\right) & =0 \\
G\left(\frac{1}{2} \Omega\right) Z\left(\frac{1}{2} \Omega\right) & =q_{1}^{-\frac{1}{2}}, \\
g\left(\frac{1}{2} \Upsilon\right) Z\left(\frac{1}{2} \Upsilon\right) & =q^{-\frac{1}{2}}, \\
y\left(\frac{1}{2} \Omega\right) g\left(\frac{1}{2} \Upsilon\right) & =-i y\left(\frac{1}{2} \Upsilon\right) G\left(\frac{1}{2} \Omega\right),
\end{array}\right\}
$$

C. 
d'où l'on conclut les formules

$$
\left.\begin{array}{rl}
B^{\prime \prime} C^{\prime \prime}: A^{\prime \prime} D^{\prime \prime} & =B^{\prime} D^{\prime}: A^{\prime} C^{\prime}=C D: A B=-1 \\
A^{\prime} B^{\prime}: C^{\prime} D^{\prime}=-A^{\prime \prime} B^{\prime \prime}: C^{\prime \prime} D^{\prime \prime} & =-\mathrm{y}^{2}\left(\frac{1}{2} \Upsilon\right): Z^{2}\left(\frac{1}{2} \Upsilon\right) \\
A^{\prime \prime} C^{\prime \prime}: B^{\prime \prime} D^{\prime \prime} & =-A^{\prime} C^{\prime}: B^{\prime} D^{\prime}=\mathrm{y}^{2}\left(\frac{1}{2} \Omega\right): Z^{2}\left(\frac{1}{2} \Omega\right) \\
A D: B C & =-A^{\prime} D^{\prime}: B^{\prime} C^{\prime}=\mathrm{y}^{2}\left(\frac{1}{2} \Omega\right): G^{2}\left(\frac{1}{2} \Omega\right) \\
& =-\mathrm{y}^{2}\left(\frac{1}{2} \Upsilon\right): \mathrm{g}^{2}\left(\frac{1}{2} \Upsilon\right)
\end{array}\right\}
$$

dont nous aurons bientôt besoin.

On peut passer maintenant à ce système général de formules, où j'écris $(-)^{n}$ au lieu de $(-1)^{m}$ :

$$
\begin{aligned}
& \Theta=(-)^{m n} \epsilon^{6 x(m,-n)} q_{1}^{-\frac{1}{2} m^{2}} q^{-\frac{1}{2} n^{2}}, \\
& \text { là, comme toujours, }(m,-n)=m \Omega-n \Upsilon \text {; } \\
& \text { y }\{x+(m, n)\}=(-)^{m+n} \Theta \mathrm{y} x, \\
& \mathrm{~g}\{x+(m, n)\}=(-)^{m} \quad \Theta g x, \\
& G\{x+(m, n)\}=(-)^{n} \quad \Theta G x, \\
& Z\{x+(m, n)\}=\quad \Theta Z x ; \\
& \Phi=(-)^{n m+\frac{1}{2} n} \epsilon^{6 x(\bar{m},-n)} q^{-\frac{1}{2} m^{2}-\frac{1}{2} m} q^{-\frac{1}{2} n^{2}} ; \\
& \text { y }\{x+(\bar{m}, n)\}=(-)^{m+n} \Phi A \mathrm{~g} x, \\
& \mathrm{~g}\{x+(\bar{m}, n)\}=(-)^{m} \quad \Phi B \mathrm{y} x, \\
& G\{x+(\bar{m}, n)\}=(-)^{n} \quad \Phi C Z x, \\
& Z\{x+(\bar{m}, n)\}=\quad \Phi D G x ; \\
& \Psi=(-)^{m n+\frac{1}{2} m} e^{6 x(m,-\bar{n})} q^{-\frac{1}{2} m^{2}} q^{-\frac{1}{2} n^{2}-\frac{1}{2} n} ; \\
& \text { y }\{x+(m, \bar{n})\}=(-)^{m+n} \Psi A^{\prime} G x, \\
& \text { g }\{x+(m, \bar{n})\}=(-)^{m} \quad \Psi B^{\prime} Z x, \\
& G\{x+(m, \bar{n})\}=(-)^{n} \quad \Psi C^{\prime} \mathbf{y} x \\
& Z\{x+(m, \bar{n})\}=\quad \Psi D^{\prime} \mathrm{g} x ; \\
& \mathrm{X}=(-)^{m n+\frac{1}{2} m+\frac{1}{2} n} e^{8 x(\bar{m},-\bar{n})} q_{1}^{-\frac{1}{2} m^{2}-\frac{1}{2} m} q^{-\frac{1}{2} n^{2}-\frac{1}{2} n} \text {; } \\
& \mathrm{y}\{x+(\bar{m}, \bar{n})\}=(-)^{m+n} \mathrm{X} A^{\prime \prime} Z x \text {, } \\
& \mathrm{g}\{x+(\bar{m}, \bar{n})\}=(-)^{m} \quad \mathrm{X} B^{\prime \prime} G x, \\
& G\{x+(\bar{m}, \bar{n})\}=(-)^{n} \quad \mathrm{X} C^{\prime \prime} \mathrm{g} x, \\
& Z\{x+(\bar{m}, \bar{n})\}=\quad \mathrm{X} D^{\prime \prime} \mathrm{y} x \text {. }
\end{aligned}
$$


Soit $x=0$ :

(G)

$$
\begin{aligned}
& \left\{\begin{array}{l}
\Theta_{0}=(-)^{m n} q_{1}^{-\frac{1}{2} m^{2}} q^{-\frac{1}{2} n^{2}} ; \\
\mathrm{y}(m, n)=0, \\
\mathrm{~g}(m, n)=(-)^{m} \Theta_{0},
\end{array} \quad \mathrm{y}^{\prime}(m, n)=(-)^{m+n} \Theta_{0},\right. \\
& G(m, n)=(-)^{n} \Theta_{0}, \\
& Z(m, n)=\quad \Theta_{0} ; \\
& \Phi_{0}=(-)^{m n+\frac{1}{2} m} q_{1}^{-\frac{1}{2} m^{2}-\frac{1}{2} m-\frac{1}{2} n^{2}} ; \\
& \mathrm{y}(\bar{m}, n)=(-)^{m+n} \Phi_{0} A \text {, } \\
& \mathrm{g}(\bar{m}, n)=\quad 0, \quad \mathrm{~g}^{\prime}(\bar{m}, n)=(-)^{m} \Phi_{0} B, \\
& G(\bar{m}, n)=(-)^{n} \quad \Phi_{0} C, \\
& Z(\bar{m}, n)=\quad \Phi_{0} D ; \\
& \Psi_{0}=(-)^{m n+\frac{1}{2} n} q_{1}^{-\frac{1}{2} m^{2}} q^{-\frac{1}{2} n^{2}-\frac{1}{2} n} ; \\
& \text { y }(m, \bar{n})=(-)^{m+n} \Psi_{0} A^{\prime}, \\
& \mathrm{g}(m, \bar{n})=(-)^{m} \quad \Psi_{0} B^{\prime}, \\
& G(m, \bar{n})=\quad 0, \quad G^{\prime}(m, \bar{n})=(-)^{n} \Psi_{0} C^{\prime}, \\
& Z(m, \bar{n})=\quad \Psi_{0} D^{\prime} ; \\
& \mathrm{X}_{0}=(-)^{m n+\frac{1}{2} m+\frac{1}{2} n} q_{1}^{-\frac{1}{2} m^{2}-\frac{1}{2} m} q^{-\frac{1}{2} n^{2}-\frac{1}{2} n} \text {; } \\
& \text { y }(\bar{m}, \bar{n})=(-)^{m+n} \mathrm{X}_{0} A^{\prime \prime} \text {, } \\
& \mathrm{g}(\bar{m}, \bar{n})=(-)^{m} \quad \mathrm{X}_{0} B^{\prime \prime} \text {, } \\
& G(\bar{m}, \bar{n})=(-)^{n} \quad X_{0} C^{\prime \prime}, \\
& Z(\bar{m}, \bar{n})=\quad 0, \quad Z^{\prime}(\bar{m}, \bar{n})=X_{0} D^{\prime \prime} .
\end{aligned}
$$

On a de suite, en prenant les différentielles des logarithmes des fonctions $J_{x}$,

$$
\left\{\begin{array}{l}
\mathrm{y}^{\prime} x: \mathrm{y} x=-B x+\boldsymbol{\Sigma}\{x-(m, n)\}^{-1}, \\
\mathrm{~g}^{\prime} x: \mathrm{g} x=-B x+\boldsymbol{\Sigma}\{x-(\bar{m}, n)\}^{-1}, \\
G^{\prime} x: G x=-B x+\boldsymbol{\Sigma}\{x-(m, \bar{n})\}^{-1}, \\
Z^{\prime} x: Z x=-B x+\boldsymbol{\Sigma}\{x-(\bar{m}, \bar{n})\}^{-1} .
\end{array}\right.
$$

( $B$ est le coefficient de $x^{2}$, dans l'exponentielle $\epsilon^{-\frac{1}{2} B x^{2}}$ des équations (B); il n'y a pas à craindre de le confondre avec le $B$ qui entre dans les équations pour y $(\bar{m}, n)$, \&c.: c'est par hasard que j'ai pris deux fois la même lettre.) 
Réduisons en fractions simples la fonction

$$
\mathrm{g} x G^{\gamma} x: \mathrm{y} x Z x .
$$

En écrivant

$$
\mathrm{g} x G x: \mathrm{y} x Z_{x}=\mathbf{\Sigma}\left[L\{x-(m, n)\}^{-1}+M\{x-(\bar{m}, \bar{n})\}^{-1}\right],
$$

on obtient

$$
\begin{aligned}
& L=\mathrm{g}(m, n) G(m, n): \mathrm{y}^{\prime}(m, n) Z(m, n)=1, \\
& M=\mathrm{g}(\bar{m}, \bar{n}) G(\bar{m}, \bar{n}): \mathrm{y}(\bar{m}, \bar{n}) Z^{\prime}(\bar{m}, \bar{n})=B^{\prime \prime} C^{\prime \prime}-A^{\prime \prime} D^{\prime \prime}=1 .
\end{aligned}
$$

Done

$$
\mathrm{g} x G x: \mathrm{y} x Z_{x}=\boldsymbol{\Sigma}\{x-(m, n)\}^{-1}-\boldsymbol{\Sigma}\{x-(\bar{m}, \bar{n})\}^{-1},
$$

c'est-à-dire

$$
\mathrm{g} x G x: \mathrm{y} x Z x=\left(\mathrm{y}^{\prime} x: \mathrm{y} x\right)-\left(Z^{\prime} x: Z x\right) .
$$

(Nous allons bientôt justifier, au moyen d'un théorème de M. Cauchy, l'emploi de cette méthode de décomposition en, fractions simples qui ne s'applique pas toujours aux fonctions transcendantes.)

On obtient de même

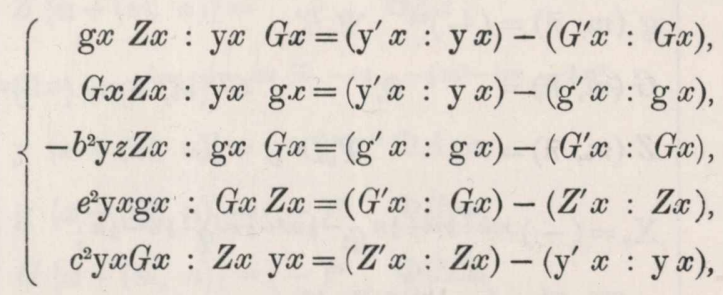

en mettant, pour abréger,

$$
\left\{\begin{array}{l}
\mathrm{y}\left(\frac{1}{2} \Upsilon\right): Z\left(\frac{1}{2} \Omega\right)=\frac{i}{e},\left({ }^{1}\right) \\
\mathrm{y}\left(\frac{1}{2} \Omega\right): Z\left(\frac{1}{2} \Omega\right)=\frac{1}{c} \\
\mathrm{y}\left(\frac{1}{2} \Omega\right): G\left(\frac{1}{2} \Omega\right)=-i \mathrm{y}\left(\frac{1}{2} \Upsilon\right) \div \mathrm{g}\left(\frac{1}{2} \Upsilon\right)=\frac{1}{b}
\end{array}\right.
$$

Donc, en éliminant les fonctions dérivées,

$$
\begin{aligned}
& G^{2} x-Z^{2} x=\quad e^{2} \mathrm{y}^{2} x, \\
& g^{2} x-G^{2} x=-b^{2} \mathrm{y}^{2} x, \\
& Z^{2} x-\mathrm{g}^{2} x=\quad c^{2} \mathrm{y}^{2} x,
\end{aligned}
$$

1 J'ai écrit $\frac{i}{e}$ au lieu de $\frac{1}{e}$ pour me conformer à la notation d'Abel; il est à peine nécessaire de remarquer que $c, e$ sont, en général, l'une et l'autre des quantités imaginaires. 
ce qui donne, en ajoutant,

$$
b^{2}=e^{2}+c^{2}, \text { ou } b=\sqrt{e^{2}+c^{2}},
$$

en nous retiendrons désormais la lettre $b$ dans cette signification. Puis

$$
\left\{\begin{array}{l}
g^{2} x=Z^{2} x-c^{2} y^{2} x, \\
G^{2} x=Z^{2} x+e^{2} y^{2} x .
\end{array}\right.
$$

Soient maintenant

$$
\left\{\begin{array}{l}
\phi x=\mathrm{y} x: Z x, \\
f x=\mathrm{g} x: Z x, \\
F x=G x: Z x .
\end{array}\right.
$$

On obtient

$(\mathrm{N})$

$$
\begin{aligned}
& \left\{\begin{array}{l}
f^{2} x=1-c^{2} \phi^{2} x, \\
F^{2} x=1+e^{2} \phi^{2} x ;
\end{array}\right. \\
& \left\{\begin{array}{l}
\phi^{\prime} x=f x F x, \\
f^{\prime} x=-c^{2} \phi x F x, \\
F^{\prime} x=e^{2} \phi x f x .
\end{array}\right.
\end{aligned}
$$

Ces équations sont précisément les équations fondarnentales d'Abel (Euvres, t. I., p. 143 [Ed. 2, p. 268]), et il en déduit

$$
\left\{\begin{array}{l}
\phi(x+y)=\frac{\phi x f y F y+\phi y f x F x}{1+e^{2} c^{2} \phi^{2} x \phi^{2} y} \\
f(x+y)=\frac{f x f y-c^{2} \phi x \phi y F x F y}{1+e^{2} c^{2} \phi^{2} x \phi^{2} y} \\
F(x+y)=\frac{F x F y+e^{2} \phi x \phi u f x f y}{1+e^{2} c^{2} \phi^{2} x \phi^{2} y}
\end{array}\right.
$$

qui sont les formules connues pour l'addition des fonctions elliptiques.

En effet, on peut écrire l'équation

$$
\phi^{\prime} x=f x F^{\prime} x
$$

sous la forme

$$
1=\frac{\phi^{\prime} x}{\sqrt{\left(1-c^{2} \phi^{2} x\right)\left(1+e^{2} \phi^{2} x\right)}},
$$

ou, en mettant $y=\phi x$,

$$
x=\int_{0}^{\phi x} \frac{d y}{\sqrt{\left(1-c^{2} y^{2}\right)\left(1+e^{2} y^{2}\right)}}
$$


on peut donc de ce point supposer connues toutes les propriétés des fonctions elliptiques. On a, par exemple,

$$
\phi\left(\frac{1}{2} \Upsilon\right)=\frac{i}{e}, \quad \phi\left(\frac{1}{2} \Omega\right)=\frac{1}{c}
$$

et de là

$$
\left\{\begin{array}{l}
\frac{1}{2} \Omega=\int_{0}^{\frac{1}{c}} \frac{d y}{\sqrt{\left(1-c^{2} y^{2}\right)\left(1+e^{2} y^{2}\right)}} \\
\frac{1}{2} \Upsilon=\int_{0}^{\frac{i}{e}} \frac{d y}{\sqrt{\left(1-c^{2} y^{2}\right)\left(1+e^{2} y^{2}\right)}}
\end{array}\right.
$$

qui déterminent $\Omega, \Upsilon$ en fonction de $c, e$. Il paraît au premier abord que $\Upsilon, \Omega$ soient des fonctions parfaitement déterminées de $c, e$. J'ai des raisons pour croire que cela n'est pas précisément le cas, et que la question admettrait des développements intéressants; mais je réserve ce sujet pour une autre occasion.

On peut, à l'aide des équations entre $\mathrm{y}\left(\frac{1}{2} \Omega\right)$, \&c., et les quantités $c$, $e$, exprimer cette suite de fonctions en termes de $c, e$. On déduit:

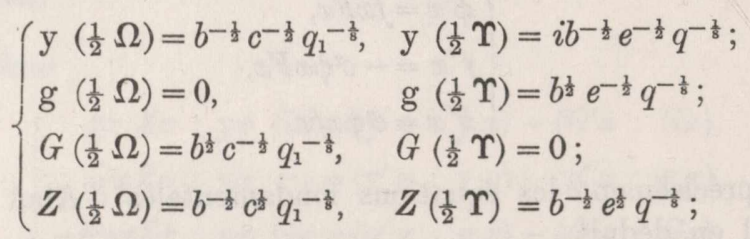

et de là

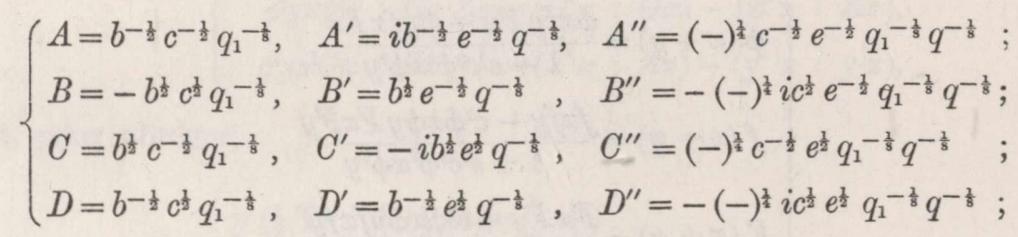

qu'on doit introduire dans toutes les formules où entrent les quantités $A, B$, \&c.

Voici le théorème de M. Cauchy (Exercices de Mathématiques, tome II. page 289):

" $\mathrm{Si}$, en attribuant au module $r$ de la variable

$$
z=r(\cos p+i \sin p)
$$

des valeurs infiniment grandes, on peut les choisir de manière que les deux fonctions

$$
\frac{f z+f(-z)}{2}, \frac{f z-f(-z)}{2 z}
$$

deviennent sensiblement nulles, quel que soit l'angle $p$, ou du moins que chacune de ces fonctions reste toujours finie ou infiniment petite, et ne cesse d'être infiniment 
petite, en demeurant finie, que dans le voisinage de certaines valeurs particulières de $p$, on aura

$$
f x=\mathcal{E} \frac{((f z))}{x-z},
$$

pourvu qu'on réduise le résidu intégral à sa valeur principale."

On se rappelle que cela veut dire qu'en supposant ces conditions satisfaites, la fonction $f x$ peut s'exprimer de la manière ordinaire comme la somme d'une suite de fractions simples, mais qu'il faut étendre d'abord la sommation aux racines de l'équation

$$
\frac{1}{f x}=0,
$$

dont les modules sont inférieurs à une certaine limite qu'on fait alors infinie.

Soit, par exemple,

$$
f x=\epsilon^{\frac{1}{2} a x^{2}+b x} \frac{T(x)}{T_{1}(x)}
$$

où $T x, T_{1} x$ ne contiennent que des facteurs qui sont des puissances entières des fonctions $\mathrm{y} x, \mathrm{~g} x, G x, Z x$. En supposant que $r$ n'est d'aucune des formes

$$
\bmod (m, n), \quad \bmod (\bar{m}, n), \quad \bmod (m, \bar{n}), \quad \bmod (\bar{m}, \bar{n}),
$$

mais, d'ailleurs, uue quantité infinie quelconque, on peut toujours écrire

$$
z=r(\cos p+i \sin p)=(m, n)+\theta
$$

où $\theta$ est une quantité finie telle qu'aucune des fonctions $\mathrm{y} \theta, \mathrm{g} \theta, G \theta, Z \theta$ ne s'évanouit, $m, n$ sont des entiers dont l'un au moins est infini, mais qui varient depuis $-\infty$ jusqu'à $\infty$, avec l'angle $p$. Soit $\gamma$ le degré de $T x, \gamma_{1}$ celui de $T_{1} x$ à l'égard de $\mathrm{y} x$, \&c.; soit aussi $\lambda=8_{1}-8$; on a, en général, une équation de cette forme,

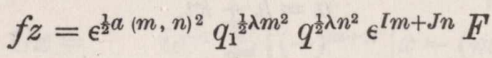

où $F$ est fini. En supposant donc que la partie réelle de

$$
a(m, n)^{2}-\lambda b \Omega^{2} m^{2}+\lambda b \Upsilon^{2} n^{2}
$$

est négative quelles que soient les valeurs de $m, n$, on a

$$
f z=0 \text {, }
$$

et de même

$$
\frac{f z+f(-z)}{2}=0, \quad \frac{f z-f(-z)}{2 z}=0 .
$$

$\mathrm{Si}$, au contraire, cette partie réelle est positive, ces trois fonctions sont toujours infinies. Il y a cependant un cas particulier à considérer, savoir, celui où cette partie réelle se réduit à $P m^{2}$ ou $Q n^{2}, P, Q$ étant des quantités positives. Il faut ici que le 
coefficient de $n$ ou de $m$ dans la partie réelle de $I m+J n$ s'évanouisse. Enfin, si les parties réelles s'évanouissent entièrement, ce qui ne peut arriver que pour

$$
a=0, \quad b=0, \quad \lambda=0,
$$

$f x$ est fini. On a donc pour $f x$ fonction impaire,

$$
\frac{f z+f(-z)}{2}=0, \quad \frac{f z-f(-z)}{2 z}=0
$$

(la seconde équation à cause du dénominateur infini $z$ ). Il est cependant certain que, dans plusieurs cas pour lesquels $f x$ est fonction paire, on peut réduire $f x$ en une suite de fractions simples: par exemple, $\mathrm{y} x: \mathrm{g} x$ est une fonction impaire que l'on peut, par ce qui précède, développer en suite de fractions simples; en écrivant $x+\frac{1}{2} \Upsilon$ au lieu de $x$, on déduit un pareil développement pour $G x: Z x$ qui est fonction paire.

Remarquons que quand la partie réelle de

$$
a(m, n)^{2}-\lambda 6 \Omega^{2} m^{2}+\lambda \odot \Upsilon^{2} n^{2}
$$

est négative pour toute valeur de $m$ ou $n$, la suite pour $f x$ est toujours convergente. En effet, les numérateurs des fractions simples contiennent ce facteur de $f z$,

$$
\epsilon^{\frac{1}{2} a(m, n)^{2}} q_{1^{\frac{1}{2} \lambda m^{2}}} q^{\frac{1}{2} \lambda n^{2}},
$$

qui s'évanouit pour les valeurs infiniment grandes de $m, n$. Dans le cas où la partie réelle est positive, le développement ne peut jamais être vrai; je crois qu'en général il est vrai, dans les cas limites, quand la suite que l'on obtient est convergente. Il y a des exceptions cependant; on en verra une en développant $\phi^{2} x$.

Avant de passer aux exemples, développons la condition pour que la partie réelle en question soit toujours négative.

En supposant

$$
a=h+k i,
$$

on obtient pour cette quantité l'expression

$$
\left.\begin{array}{rl} 
& m^{2}\left\{h\left(\omega^{2}-\omega^{\prime 2}\right)-2 k \omega \omega^{\prime}-\frac{\lambda \pi \bmod \left(\omega v^{\prime}-\omega^{\prime} v\right)}{v^{2}+v^{\prime 2}}\right\} \\
+ & n^{2}\left\{h\left(v^{2}-v^{\prime 2}\right)-2 k v v^{\prime}-\frac{\lambda \pi \bmod \left(\omega v^{\prime}-\omega^{\prime} v\right)}{\omega^{2}+\omega^{\prime 2}}\right\} \\
+ & 2 m n\left\{h\left(\omega v-\omega^{\prime} v^{\prime}\right)-k\left(\omega v^{\prime}+\omega^{\prime} v\right)\right\},
\end{array}\right\}
$$

qui doit toujours rester négative. Cela donne, après quelques réductions, la condition

$$
\left.\begin{array}{rl}
h^{2}+k^{2} & +\frac{2 \lambda \pi\left(\omega v+\omega^{\prime} v^{\prime}\right)}{\left(\omega^{2}+\omega^{\prime 2}\right)\left(\varpi^{2}+\omega^{\prime 2}\right) \bmod \left(\omega v^{\prime}-\omega^{\prime} v\right)}\left[\left(\omega v-\omega^{\prime} v^{\prime}\right) h+\left(\omega v^{\prime}+\omega^{\prime} v\right) k\right] \\
& -\frac{\lambda^{2} \pi^{2}}{\left(\omega^{2}+\omega^{\prime 2}\right)\left(v^{2}+v^{\prime 2}\right)}<0 .
\end{array}\right\}
$$


Les valeurs

$$
h=0, \quad k=0
$$

satisfont à cette condition, laquelle du reste (en considérant $h, k$ comme les coordonnées d'un point) est satisfaite pour tout point situé en dedans d'un certain cercle qui inclut l'origine, et dont on aurait l'équation en remplaçant le signe $<$ par le signe $=$ dans la formule (38).

On obtient de cette façon une grande variété de formules particulières. Par exemple celles-ci :

(U)

$$
\begin{aligned}
& \left(\epsilon^{\frac{1}{2} a x^{2}+b x}: \mathrm{y} x=\quad \boldsymbol{\Sigma}\left[(-)^{-m n-m-n} \epsilon^{\frac{1}{3} a(m, n)^{2}+b(m, n)} q_{1}^{\frac{1}{2} m^{2}} q^{\frac{3}{n^{2}}}\{x-(m, n)\}^{-1}\right],\right.
\end{aligned}
$$

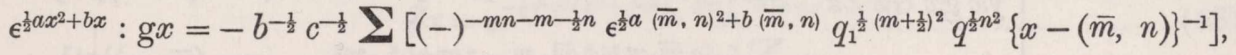

$$
\begin{aligned}
& \epsilon^{\frac{3}{2} a x^{2}+b x}: G x=i b^{-\frac{1}{2}} c^{-\frac{1}{2}} \mathbf{\Sigma}\left[(-)^{-m n-\frac{1}{2} m-n} e^{\frac{3}{2} a(m, \bar{n})^{2}+b(m, \bar{n})} q_{1^{\frac{1}{2} m^{2}}} q^{\frac{1}{2}\left(n+\frac{1}{2}\right)^{2}}\{x-(m, \bar{n})\}^{-1}\right], \\
& \epsilon^{\frac{3}{3} a x^{2}+b x}: Z x=i c^{-\frac{1}{2}} e^{-\frac{1}{2}} \quad \Sigma\left[(-)^{-\left(m+\frac{1}{2}\right)\left(n+\frac{1}{2}\right)} \epsilon^{\frac{1}{2} a(\bar{m}, \bar{n})^{2}+b(\bar{m}, \bar{n})} q_{1^{\frac{1}{2}}\left(m+\frac{1}{2}\right)^{2}} q^{\frac{1}{2}\left(n+\frac{1}{2}\right)^{2}}\{x-(\bar{m}, \bar{n})\}^{-1}\right],
\end{aligned}
$$

dans lesquelles, pour trouver les limites de $a$, il faut faire $\lambda=1$ dans la formule (38).

On a ensuite ce système de formules sans exponentielles,

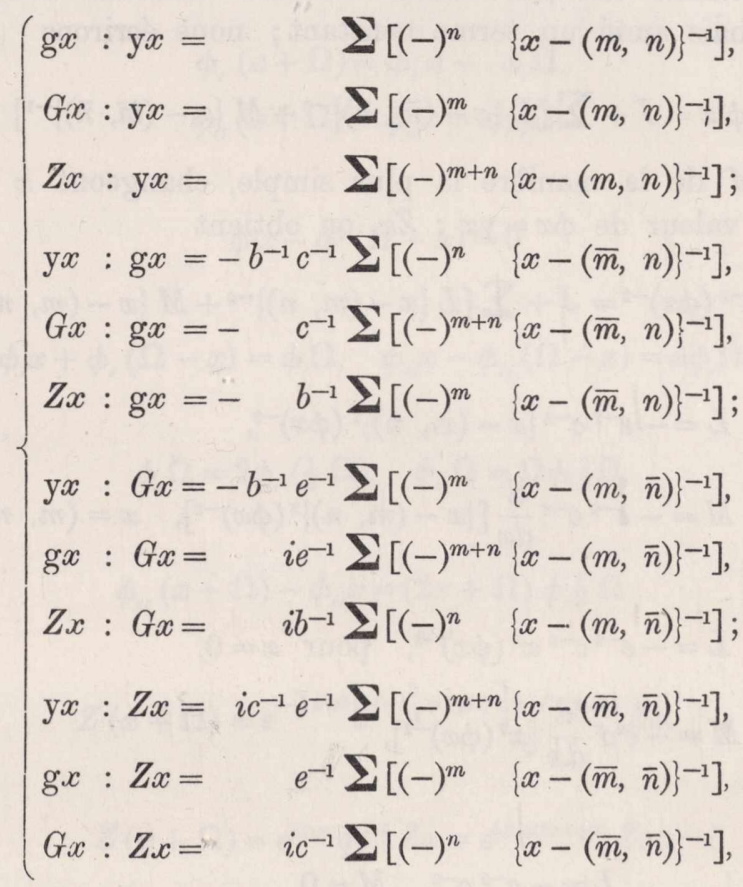

dont quelques-unes ont été données par Abel. Il faut remarquer que celles de ces équations où la fonction est impaire sont justifiées par le théorème de $\mathrm{M}$. Cauchy, et que les autres se déduisent de celles-ci. On peut trouver de même le développement des fonctions

$$
\frac{1}{\mathrm{y} x \mathrm{~g} x}, \ldots, \quad \frac{G x}{\mathrm{y} x \mathrm{~g} x}, \ldots, \quad \frac{\mathrm{y} x \mathrm{~g} x}{Z x G x}
$$

C. 
(celles-ci, qui sont toutes impaires, ont été déja considérées; nous venons de faire voir que le développement est admissible);

$$
\frac{1}{\mathrm{y} x \mathrm{~g} x G x}, \ldots, \quad \frac{Z x}{\mathrm{y} x \mathrm{~g} x G x}, \ldots, \quad \frac{1}{\mathrm{y} x \mathrm{~g} x Z x}, \ldots,
$$

chacune, excepté celles de la suite $\frac{\mathrm{y} x \mathrm{~g} x}{G x Z x}$, multipliée par un facteur exponentiel $e^{\natural a x^{2}+b x}$. Par exemple,

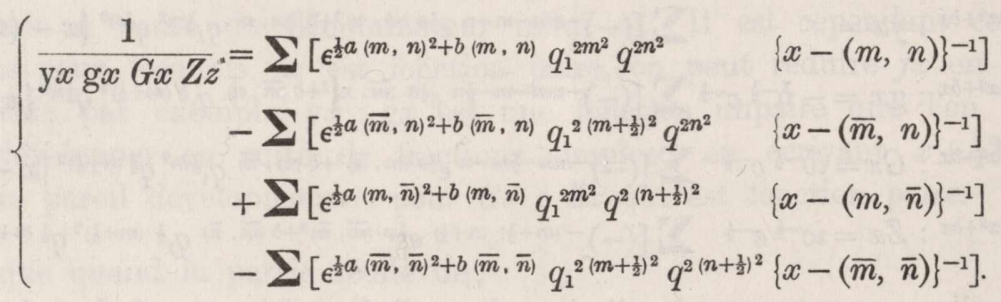

Mais on est conduit à un résultat beaucoup plus important en considérant, par exemple, le développement de $\phi^{2} x$. Cette fonction contient non-seulement une suite de fractions simples, màis aussi un terme constant; nous écrirons

$$
\phi^{2} x=J+\boldsymbol{\Sigma}\left[L\{x-(\bar{m}, \bar{n})\}^{-2}+M\{x-(\bar{m}, \bar{n})\}^{-1}\right]
$$

Pour déterminer $L, M$ de la manière la plus simple, changeons $x$ en $x+\frac{1}{2} \Omega+\frac{1}{2} \Upsilon$. En faisant attention à la valeur de $\phi x=\mathrm{y} x: Z x$, on obtient

$$
-e^{-2} c^{-2}(\phi x)^{-2}=J+\mathbf{\Sigma}\left[L\{x-(m, n)\}^{-2}+M\{x-(m, n)\}^{-1}\right]
$$

de là

$$
\begin{aligned}
& L=-e^{-2} c^{-2}\{x-(m, n)\}^{2}(\phi x)^{-2}, \\
& M=-e^{-2} c^{-2} \frac{d}{d x}\left[\{x-(m, n)\}^{2}(\phi x)^{-2}\right], \quad x=(m, n),
\end{aligned}
$$

ou enfin

$$
\begin{aligned}
& L=-e^{-2} c^{-2} x^{2}(\phi x)^{-2}, \text { pour } x=0 \\
& M=-e^{2} c^{2} \frac{d}{d x}\left[x^{2}(\phi x)^{-2}\right]
\end{aligned}
$$

ce qui donne

$$
\left.\begin{array}{rl}
L & =-e^{-2} c^{-2}, \quad M=0, \\
\phi^{2} x & =J-e^{-2} c^{-2} \mathbf{\Sigma}\{x-(\bar{m}, \bar{n})\}^{-2} .
\end{array}\right\}
$$

En intégrant deux fois

$$
\int_{0}^{x} d x \int_{0}^{x} \phi^{2} x d x=\frac{1}{2} J x^{2}+e^{-2} c^{-2} \mathbf{\Sigma} \log \{x-(\bar{m}, \bar{n})\}
$$


ou, à cause de $\log Z x=-\frac{1}{2} B x^{2}+\sum \log \{x-(\bar{m}, \bar{n})\}$,

$$
\int_{0}^{x} d x \int_{0}^{x} \phi^{2} x d x=\frac{1}{2} I x^{2}+e^{-2} c^{-2} \log Z x .
$$

en mettant, pour abréger,

$$
I=J-e^{-2} c^{-2} B
$$

(on u'a pas ajouté de constante arbitraire, parce que $Z x$ est fonction paire de $x$ qui se réduit à l'unité pour $x=0$ ). Puis, on a

$$
Z x=\epsilon^{-\frac{1}{2} e^{2} c^{2} I x^{2}+e^{2} c^{2} \int_{0}^{x} d x \int_{0}^{x} \phi^{2} x d x}
$$

De là il est facile de déterminer la valeur de $I$. Soit, pour un moment,

$$
\phi, x=\int_{0}^{x} \phi^{2} x d x, \quad \phi_{11} x=\int_{0}^{x} \phi, x d x
$$

Puisque

$$
\phi^{2}(x+\Omega)-\phi^{2} x=0
$$

on a

$$
\begin{aligned}
& \phi_{1}(x+\Omega)=\phi_{1} x-\phi, \Omega \\
& \phi_{1 /}(x+\Omega)-\phi_{11} x=x \phi_{1} \Omega .
\end{aligned}
$$

Mais de l'équation

$$
\phi^{2} x-\phi^{2}(\Omega-x)=0
$$

on déduit

$$
\phi_{,} x+\phi_{,}(\Omega-x)=\phi_{,} \Omega, \quad \phi_{1} x-\phi_{11}(\Omega-x)=x \phi_{,} \Omega,
$$

ou, en faisant $x=\frac{1}{2} \Omega$,

$$
\phi, \Omega=2 \phi,\left(\frac{1}{2} \Omega\right), \quad \phi_{1 /} \Omega=\Omega \phi_{, 2}, \frac{1}{2},
$$

c'est-à-dire

$$
\phi_{11}(x+\Omega)-\phi_{11} x=(2 x+\Omega) \phi_{, 2} \frac{1}{2} \Omega
$$

et de là

$$
Z(x+\Omega)=\epsilon^{-\frac{1}{2} e^{2} c^{2}\left[I-\frac{2}{\Omega} \phi\left(\frac{1}{2} \Omega\right)\right] 2\left(\Omega x+\Omega^{2}\right)} Z x .
$$

Mais on a déjà

$$
Z(x+\Omega)=\epsilon^{\beta \Omega x} q_{1}^{-\frac{1}{2}} Z x=\epsilon^{\frac{1 \beta}{2}\left(2 \Omega x+\Omega^{2}\right)} Z x ;
$$

donc enfin

$$
-e^{2} c^{2}\left[I-\frac{2}{\Omega} \phi,\left(\frac{1}{2} \Omega\right)\right]=\beta
$$

c'est-à-dire

$$
-\frac{1}{2} e^{2} c^{2} I=\frac{1}{2} \beta-\frac{e^{2} c^{2}}{\Omega} \phi_{1}\left(\frac{1}{2} \Omega\right)=\frac{1}{2} \beta-\frac{e^{2} c^{2}}{\Omega} \int_{0}^{\frac{1}{2} \Omega} \phi^{2} x d x
$$


Soit, pour abréger,

$$
M=\frac{e^{2} c^{2}}{\Omega} \int_{0}^{\frac{1}{3} \Omega} \phi^{2} x d x
$$

on a cette équation,

$$
Z x=\epsilon^{\left(\frac{1}{2} \beta-M\right) x^{2}+e^{2} c^{2}} \int_{0}^{x} d x \int_{0}^{x} \phi^{2} x d x,
$$

qui exprime la fonction $Z x$ au moyen de $\phi x$. C'est, en effet, la formule remarquable de M. Jacobi, que nous avons citée dans l'introduction de ce Mémoire.

En changeant seulement la notation, on a, d'après M. Jacobi,

$$
\begin{aligned}
& \phi^{2}(x+a)-\phi^{2}(x-a)=\frac{4(\phi a f a F a \phi x f x F x)}{\left(1+e^{2} c^{2} \phi^{2} a \phi^{2} x\right)^{2}} . \\
& \int_{0}^{x}\left\{\phi^{2}(x+a)-\phi^{2}(x-a)\right\} d x=\frac{2 \phi a f a F a \phi^{2} x}{1+e^{2} c^{2} \phi^{2} a \phi^{2} x}
\end{aligned}
$$

ou, ce qui est la même chose,

$$
\int_{-a}^{x} \phi^{2}(x+a) d x-\int_{a}^{x} \phi^{2}(x-a) d x-2 \int_{0}^{x} \phi^{2} a d a=\frac{2 \phi a f a F a \phi^{2} x}{1+e^{2} c^{2} \phi^{2} a \phi^{2} x} \ldots \ldots
$$

De là, en multipliant par $e^{2} c^{2}$, et faisant attention à la valeur de $Z x$,

$$
\frac{Z^{\prime}(x+a)}{Z(x+a)}-\frac{Z^{\prime}(x-a)}{Z(x-a)}-\frac{2 Z^{\prime} a}{Z a}=\frac{2 e^{2} c^{2} \phi a f a F a \phi^{2} x}{1+e^{2} c^{2} \phi^{2} a \phi^{2} x}
$$

Écrivant dans cette équation $a, x$ au lieu de $x$, $a$, et ajoutant, on obtient

$$
\frac{Z^{\prime} x}{Z x}+\frac{Z^{\prime} a}{Z a}-\frac{Z^{\prime}(x+a)}{Z(x+a)}=e^{2} c^{2} \phi a \phi x \phi(a+x)
$$

En intégrant la même équation par rapport à $a$,

$$
\left.\begin{array}{rl}
\log Z(x+a) & +\log Z(x-a)-2 \log Z x-2 \log Z a \\
& =\log \left(1+e^{2} c^{2} \phi^{2} a \phi^{2} x\right)
\end{array}\right\}
$$

c'est-à-dire

$$
Z(x+a) Z(x-a)=Z^{2} x Z^{2} a\left(1+e^{2} c^{2} \phi^{2} a \phi^{2} x\right) .
$$

ou, ce qui est la même chose,

$$
Z(x+a) Z(x-a)=Z^{2} x Z^{2} a+e^{2} c^{2} y^{2} x y^{2} a
$$

de laquelle on déduit facilement, en écrivant $x+\frac{1}{2} \Omega, x+\frac{1}{2} \Upsilon, x+\frac{1}{2} \Omega+\frac{1}{2} \Upsilon$ au lieu de $x$, les équations complémentaires

$$
\text { (Y) .................... }\left\{\begin{array}{l}
\mathrm{y}(x+a) \mathrm{y}(x-a)=\mathrm{y}^{2} x Z^{2} a-\mathrm{y}^{2} a Z^{2} x, \\
\mathrm{~g}(x+a) \mathrm{g}(x-a)=\mathrm{g}^{2} x Z^{2} a-c^{2} \mathrm{~g}^{2} a Z^{2} x, \\
G(x+a) G(x-a)=G^{2} x Z^{2} a+e^{2} G^{2} a Z^{2} x .
\end{array}\right.
$$


Quoiqu'elle ne soit pas liée très-étroitement avec la théorie actuelle, on peut ajouter ici cette autre formule de M. Jacobi, qu'il obtient en intégrant par rapport à $x$, au lieu de $a$,

$$
\Pi(x, a)=\int_{0}^{x} \frac{-e^{2} c^{2} \phi a f f a F a \phi^{2} x d x}{1+e^{2} c^{2} \phi^{2} a \phi^{2} x}=\frac{1}{2} \log \frac{Z(x-a)}{Z(x+a)}+x \frac{Z^{\prime} a}{Z a} \ldots \ldots \ldots
$$

au moyen de laquelle il déduit des formules pour l'addition des arguments ou des paramètres des fonctions de la troisième espèce. On trouve aussi, dans les Fund. Nova, quelques formules déduites de l'équation (49) pour exprimer $\frac{Z(x-a) Z(y-a) Z(x+y+a)}{Z(x+a) Z(y+a) Z(x+y-a)}$ au moyen de la fonction $\phi$; il serait facile de déduire des équations semblables pour les autres fonctions $\mathrm{y}, \mathrm{g}, G$.

\section{Note sur une intégrale définie.}

Soient $k_{1}, k$ des quantités réelles dont la première est la plus grande; $\Omega=\omega+\omega^{\prime} i$, $\Upsilon=v+v^{\prime} i$ des quantités quelconques, telles que $\omega v^{\prime}-\omega^{\prime} v$ ne s'évanouisse pas, et écrivons

$$
u=\int_{k}^{k_{1}} \frac{d x}{\Omega+\Upsilon x}
$$

Lintégrale $u$ a toujours une valeur finie et déterminée, puisque le dénominateur ne devient jamais zéro. On a évidemment

$$
u=\int_{0}^{\infty} d x\left[\frac{1}{\Omega+\Upsilon(x+k)}-\frac{1}{\Omega+\Upsilon\left(x+k_{1}\right)}\right] .
$$

et l'intégrale indéfinie est

$$
u=-\frac{1}{\Upsilon} \log \left\{ \pm \frac{\Omega+\Upsilon(x+k)}{\Omega+\Upsilon\left(x+k_{1}\right)}\right\}
$$

il faut passer de là à l'intégrale définie, entre les limites $0, \infty$. Soit

$$
\frac{\Omega+\Upsilon(x+k)}{\Omega+\Upsilon\left(x+k_{1}\right)}=A_{x}+i B_{x}
$$

Il est facile de voir qu'en faisant abstraction d'un dénominateur toujours positif, $A_{x}$ est une fonction du second degré en $x$, et $B_{x}$ se réduit à la quantité constante $\left(\omega v^{\prime}-\omega^{\prime} v\right)\left(k_{1}-k\right)$. Le signe de $B_{x}$ est donc toujours le même que celui de $\omega v^{\prime}-\omega^{\prime} v$; quant à celui de $A_{x}$, puisque évidemment $A_{\infty}=1$, il est clair que si $A_{0}$ est positif, $A_{x}$ reste toujours positif, ou change deux fois de signe quand $x$ passe de 0 à $\infty$. Si, au contraire, $A_{0}$ est négatif, $A_{x}$ est négatif depuis $x=0$ jusqu'à une certaine valeur positive de $x, x=\alpha$, et positif depuis $x=\alpha$ jusqu'à $x=\infty$. Considérons d'abord ce dernier cas. En représentant par $L x$ la valeur principale de log $x$ (cela suppose que la partie réelle de $x$ est positive), on obtient cette valeur de $u$,

$$
u=\frac{1}{\Upsilon} L\left[-\frac{\Omega+\Upsilon k_{1}}{\Omega+\Upsilon k}\right]-\frac{1}{\Upsilon} L\left[-\frac{\Omega+\Upsilon\left(\alpha+k_{1}-\epsilon\right)}{\Omega+\Upsilon(\alpha+k-\epsilon)}\right]-\frac{1}{\Upsilon} L\left[\frac{\Omega+\Upsilon\left(\alpha+k_{1}+\epsilon\right)}{\Omega+\Upsilon(\alpha+k+\epsilon)}\right],
$$


où ₹ est supposé une quantité infiniment petite positive. La somme des derniers termes se réduit à

$$
\frac{i}{\Upsilon}\left(-\arctan \frac{B_{a}}{A_{a-\epsilon}}+\arctan \frac{A_{a}}{B^{a+\epsilon}}\right)
$$

où, comme à l'ordinaire, $\arctan x$ doit être situé entre les limites $\pm \frac{1}{2} \pi$.

Dans cette formule, $A_{a-e}$ est une quantité infiniment petite et négative; $A_{a+e}$ est une quantité infiniment petite et positive; donc, quand $B_{\alpha}$ est négatif, les arcs se réduisent à $\frac{1}{2} \pi,-\frac{1}{2} \pi$, et si $B_{a}$ est positif, à $-\frac{1}{2} \pi, \frac{1}{2} \pi$. On a donc

$$
u=\frac{1}{\Upsilon} L\left(-\frac{\Omega+\Upsilon k_{1}}{\Omega+\Upsilon k}\right) \pm \frac{1}{\Upsilon} \pi i
$$

où il faut se servir du signe supérieur ou inférieur selon que $\omega v^{\prime}-\omega^{\prime} v$ est positif ou négatif. Dans le cas où $A_{0}$ est positif, si $A_{x}$ reste toujours positif, on obtient tout de suite

$$
u=\frac{1}{\Upsilon} L\left(\frac{\Omega+\Upsilon k_{1}}{\Omega+\Upsilon k}\right)
$$

Si $A_{x}$ change deux fois de signe, par exemple pour $x=\alpha$ et $x=6$, il faut introduire les corrections

$$
\frac{i}{\Upsilon}\left(-\arctan \frac{B_{a}}{A_{a-\epsilon}}+\arctan \frac{B_{a}}{A_{a+\epsilon}}\right)+\frac{i}{\Upsilon}\left(-\arctan \frac{B_{\varepsilon}}{A_{6-\epsilon}}+\arctan \frac{B_{\varepsilon}}{A_{6+\epsilon}}\right),
$$

qui se détruisent l'une l'autre, en sorte que l'on a le même résultat que si $A_{x}$ était toujours positif. En se servant de la notation employée dans le Mémoire, on a dans tous les cas

$$
u=L_{ \pm \pi i}\left(\frac{\Omega+\Upsilon k_{1}}{\Omega+\Upsilon k}\right)
$$

où le signe se détermine d'après celui de $\omega v^{\prime}-\omega^{\prime} v$.

En particulier,

ou

$$
\begin{gathered}
\int_{-k}^{k} \frac{d x}{\Omega+\Upsilon x}=\Omega \int_{0}^{k} \frac{d x}{\Omega^{2}-\Upsilon^{2} x^{2}}=\frac{1}{\Upsilon} L_{ \pm \pi i}\left(\frac{\Omega+\Upsilon k}{\Omega-\Upsilon k}\right) \\
\int_{0}^{\tan a} \frac{d x}{\Omega^{2}-\Upsilon^{2} x^{2}}=\frac{1}{2 \Omega \Upsilon} L_{ \pm \pi i}\left(\frac{\Omega+\Upsilon \tan a}{\Omega-\Upsilon \tan \alpha}\right)
\end{gathered}
$$

Je ne sais pas si l'on a cherché avant moi la valeur exacte de cette intégrale définie, qui est cependant la plus simple qu'on puisse imaginer, et qui devrait, je crois, trouver place dans les livres élémentaires.

NoтA. Une partie de ces recherches a été déjà imprimée dans le Cambridge Mathematical Journal [24]; je me suis borné au cas où $\Omega, \Upsilon$ sont de la forme $\omega$, vi, ce qui simplifie beaucoup la détermination des intégrales définies doubles; mais la forme générale des résultats en est très-peu affectée. 